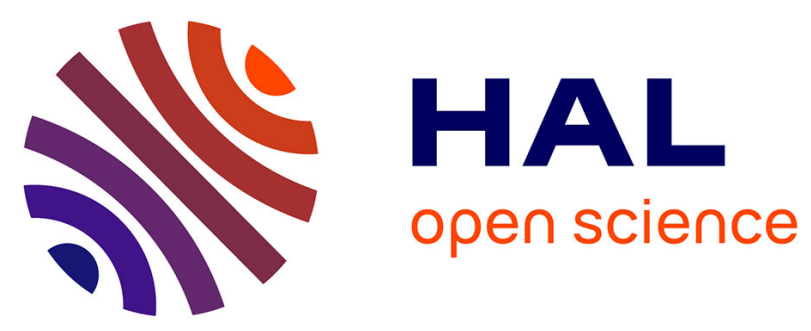

\title{
Mode transitions in distributed Bragg reflector semiconductor lasers: experiments, simulations and analysis
}

M Radziunas, K.-H Hasler, B Sumpf, Tran Quoc Tien, H Wenzel

\section{- To cite this version:}

M Radziunas, K.-H Hasler, B Sumpf, Tran Quoc Tien, H Wenzel. Mode transitions in distributed Bragg reflector semiconductor lasers: experiments, simulations and analysis. Journal of Physics B: Atomic, Molecular and Optical Physics, 2011, 44 (10), pp.105401. 10.1088/0953-4075/44/10/105401 . hal-00617388

\section{HAL Id: hal-00617388 \\ https://hal.science/hal-00617388}

Submitted on 28 Aug 2011

HAL is a multi-disciplinary open access archive for the deposit and dissemination of scientific research documents, whether they are published or not. The documents may come from teaching and research institutions in France or abroad, or from public or private research centers.
L'archive ouverte pluridisciplinaire HAL, est destinée au dépôt et à la diffusion de documents scientifiques de niveau recherche, publiés ou non, émanant des établissements d'enseignement et de recherche français ou étrangers, des laboratoires publics ou privés. 


\title{
Mode transitions in DBR semiconductor lasers: Experiments, simulations and analysis
}

\author{
M. Radziunas ${ }^{1}$, K.-H. Hasler ${ }^{2}$, B. Sumpf ${ }^{2}$, \\ Tran Quoc Tien ${ }^{2,3}$, and H. Wenzel ${ }^{2}$ \\ 1 Weierstrass Institute, Mohrenstr. 39, 10117 Berlin, Germany. \\ E-mail: radziuna@wias-berlin.de \\ 2 Ferdinand-Braun-Institut, Leibniz-Insitut für Höchstfrequenztechnik, \\ Gustav-Kirchhoff-Str. 4, 12489 Berlin, Germany. \\ E-mail: hasler@fbh-berlin.de, \\ bernd.sumpf@fbh-berlin.de,wenzel@fbh-berlin.de \\ 3 Vietnamese Academy of Science and Technology, 18 Hoang Quoc Viet, Cau \\ Giay, Hanoi, Vietnam. \\ E-mail: tientq@ims.vast.ac.vn
}

\begin{abstract}
The performance of a multisection DBR semiconductor laser emitting around $1.06 \mu \mathrm{m}$ is experimentally and theoretically investigated. A thermal-induced change of the refractive index implied by an increase of the injection current yields nearly-periodic transitions between neighboring cavity modes. These transitions are explained by means of a modal analysis and and by numerical simulations based on the traveling wave model.
\end{abstract}

PACS numbers: 42.55.Px, 42.65.bc, 42.60.Pk, 42.60.Mi

Submitted to: J. Phys. B: At. Mol. Opt. Phys.

\section{Introduction}

Wavelength stabilized single-lateral mode semiconductor lasers are required for many applications such as frequency conversion, free-space communication, spectroscopy and metrology. One possibility to achieve wavelength stabilization is through the integration of a Bragg grating into the semiconductor chip. The resulting distributed feedback (DFB) or distributed Bragg reflector (DBR) lasers emit several hundreds of milliwatts and are ideally suited for the above mentioned applications [1, 2].

DFB lasers are typically single-section devices with the Bragg grating extending over the whole amplifying cavity. In order to facilitate operation in the same longitudinal mode over a large power range, the front facet is anti-reflection coated to suppress side modes. However, this leads to a high sensitivity to feedback from external surfaces and does not prevent possible transitions between the two DFB resonance modes.

DBR lasers consist of a reflector section containing the Bragg grating and one or several amplifying sections. Fig. 1 shows a schematics of a DBR laser. In order to form 


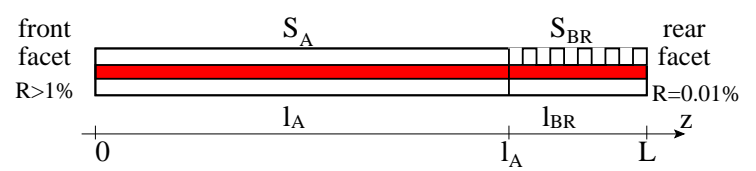

Figure 1. Scheme of a DBR semiconductor laser consisting of an amplifying section $S_{A}$ and a DBR section $S_{B R}$.

a resonator, the front facet has a reflectivity of $R \geq 1 \%$ with the value depending on the length of the amplifying sections. Due to the fact that all sections are separately driven, DBR lasers exhibit periodic undulations in the power-current characteristics and temporal instabilities owing to longitudinal mode hopping. On the other hand, compared to DFB lasers they are more robust to external perturbations and can be more cost-effectively fabricated due to the utilization of surface Bragg gratings $[3,4,5]$. Therefore, it is of great practical interest to reduce the modal instabilities which often hinder the applicability of DBR lasers.

The main aim of our paper is to use a theoretical analysis of the mode-hopping phenomena in DBR lasers to gain a better insight into the underlying physics. Our mathematical model is given by the traveling wave (TW) equations for the complex slowly varying amplitudes of the counter-propagating optical fields, coupled to equations for the induced polarization functions and the excess carrier density [6]. Following [7, 8], we relate the mode-hopping in DBR lasers with temperature induced shifts of the DBR stopband and of full cavity resonances. In the considered TW model, these thermal effects are represented by a linear nonlocal dependence of the refractive index on the inhomogeneous injection current [9].

Our explanation of the mode transitions is based on observations and analysis of instantaneous longitudinal optical modes [10] and their dynamics in edge-emitting multisection lasers $[9,11,12]$. According to our inhomogeneous heating model, the injection currents affect the refractive indices of separate laser parts differently and thereby imply a relative shift of the cavity modes with respect to the reflectivity of the DBR section. This relative shift is responsible for the transitions between modes, observable both in optical experiments and in simulations.

Our paper is organized as follows. In Section 2 we give a short description of the laser device and present some measured characteristics. After introducing the mathematical model in Section 3, we give a definition of optical modes and analyze the relationship between them as well as their dependence on model parameters in Section 4. Section 5 is devoted to the discussion of numerical simulation results. Finally, some conclusions are drawn in Section 6.

\section{Experiments}

The DBR lasers under investigation consist of a $3 \mathrm{~mm}$ long amplifying section $S_{A}$ and a $1 \mathrm{~mm}$ long DBR section $S_{B R}$ (see Fig. 1). The DBR section consists of a sixth order Bragg grating with a period of about $1 \mu \mathrm{m}$ that was defined by an i-line wafer stepper and dry etched into the surface of epitaxial layer structure [3]. It can not be electrically contacted. The effective reflection coefficient of the Bragg grating is about $55 \%$. The front and rear facets were coated with dielectric layers to obtain reflectivities of $1 \%$ and $\approx 0.01 \%$, respectively. Fundamental lateral mode operation is ensured by the use of a $5 \mu \mathrm{m}$ wide ridge waveguide. The active region consisting of three quantum wells 
with a thickness of $7 \mathrm{~nm}$ is located asymmetrically in a super large optical cavity and extends over the whole cavity. More details on the layer structure can be found in [13] and $[14]$.

Experimental characteristics of an example DBR laser driven in continuous-wave mode are presented in Figs. 2 and 3. All these figures indicate nearly-periodic changes of the lasing states as a function of the current $I_{A}$ injected into the amplifying section. Fig. 2 shows the emitted optical power at the front and rear facets versus $I_{A}$. The threshold current is $90 \mathrm{~mA}$. At the front facet the power-current characteristic is nearly linear with a slope efficiency of $0.75 \mathrm{~W} / \mathrm{A}$ up to the power of $600 \mathrm{~mW}$, except at periodically reappearing jumps.

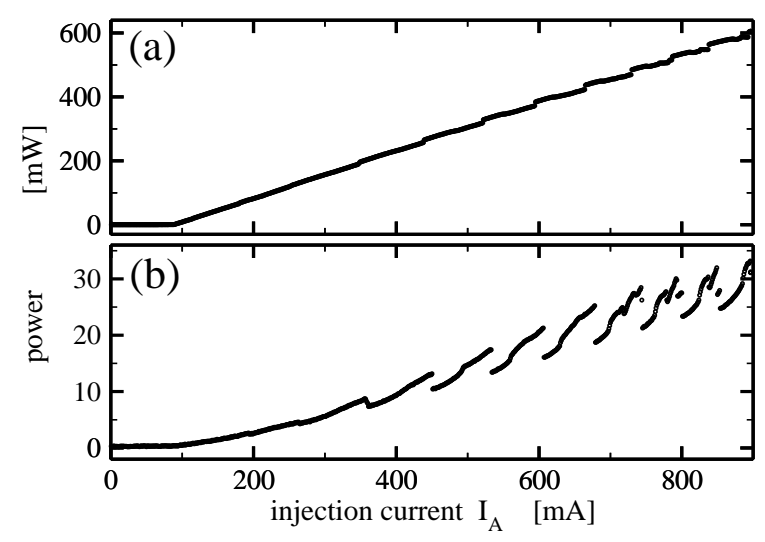

Figure 2. Power-current characteristics of the DBR laser measured at the front (a) and rear (b) facets.

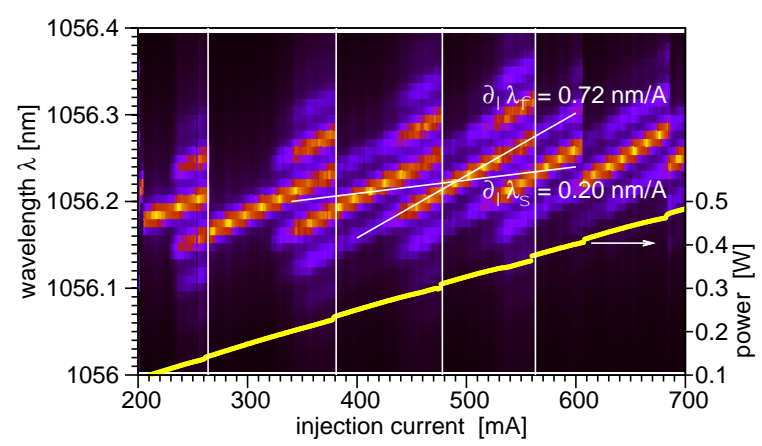

Figure 3. Representation of the measured optical spectra (mapping) and the emitted power (light curve) at the front facet of the laser as a function of the increased injection current. White slanted lines indicate the estimated slow $\left(\partial_{I} \lambda_{s}\right)$ and fast $\left(\partial_{I} \lambda_{f}\right)$ red shifts of the lasing wavelength, compare (6) and (8).

We note, that while during these jumps the power emitted at the front facet increases (panel a), the power emitted at the rear facet decreases (panel b). A nonsmooth increase of the power before each jump (most visible at the rear facet of the device) can be explained in terms of a non-stationary laser emission.

This non-stationary lasing involving two or more optical modes can be also recognized in Fig. 3 where an evolution of the optical spectrum with the increase of $I_{A}$ 
is shown. Here, each injection current "period" determined by a pair of neighboring vertical white lines contains the following typical operation regimes.

- At the low-current side of each period the laser shows a single wavelength emission.

- With an increase of $I_{A}$ several neighboring spectral peaks start to grow indicating the formation of periodic field intensity pulsations [11].

- At the high-current side of each period an abrupt change of the spectrum and field intensities occur.

- After such a jump the laser operates once more a single wavelength now at the neighboring short-wavelenght optical mode.

The step-like red shift of the spectral peaks within each period is due to a limited spectral resolution of our measuring equipment. A $\sim 45 \mathrm{pm}$ separation of the neighboring spectral peaks corresponds to the spacing of the adjacent laser modes. The periodicity of the mode jumps, the averaged slow and periodic fast red shifts of the lasing wavelength (indicated by the slanted white lines in Fig. 3) as well as an increase or decrease of the field emission from both laser facets during the mode jumps will be discussed below in this paper.

\section{Traveling wave model}

To simulate and analyze the dynamics of the DBR laser we use the traveling wave (TW) model [6]. It governs the temporal-spatial evolution of the complex slowly varying counter-propagating optical fields $E^{ \pm}(z, t)$ (scaled so that $|E|^{2}=\left|E^{+}\right|^{2}+\left|E^{-}\right|^{2}$ is the local photon density) and the induced polarization functions $p^{ \pm}(z, t)$ :

$$
\begin{aligned}
& \frac{n_{g}}{c_{0}} \partial_{t} E^{ \pm}=\left(\mp \partial_{z}-i \beta(n, I)-\mathcal{D}\right) E^{ \pm}-i \kappa E^{\mp}+\mathcal{F}_{s p}^{ \pm}, \\
& \text {b.c. }: E^{+}(0, t)=r_{0} E^{-}(0, t), E^{-}(L, t)=r_{L} E^{+}(L, t), \\
& \mathcal{D} E^{ \pm}=\frac{\bar{g}}{2}\left(E^{ \pm}-p^{ \pm}\right), \quad \partial_{t} p^{ \pm}=\bar{\gamma}\left(E^{ \pm}-p^{ \pm}\right)+i \bar{\omega} p^{ \pm} .
\end{aligned}
$$

Here, $t$ denotes time, $z \in[0, L]$ corresponds to the longitudinal propagation direction, $\mathcal{F}^{ \pm}$represents the spontaneous emission, $r_{0}$ and $r_{L}$ are the complex reflectivities of the field amplitudes at the front and rear facets. The parameters $\bar{g}, \bar{\gamma}$ and $\bar{\omega}$ denote the amplitude, the half width and the peak frequency of Lorentzian approximation of the material gain profile [6], $n_{g}$ is the group index, and $\kappa$ is the field coupling coefficient which is different from zero only in the DBR section $S_{B R}$.

The field equations (1) are coupled to the rate equations for the real excess carrier density $n(z, t)$ :

$$
\begin{aligned}
\partial_{t} n(z, t)= & \frac{J(z)}{e \sigma}-\left(A n+B n^{2}+C n^{3}\right)- \\
& -\frac{c_{0}}{n_{g}} \Re e\left(\sum_{\nu= \pm} E^{\nu *}(g(n)-2 \mathcal{D}) E^{\nu}\right), \\
\left.J(z)\right|_{z \in S_{k}}= & \frac{I_{k}}{l_{k}}, \quad k \in\{A, B R\} .
\end{aligned}
$$

Here, $J(z)$ is the injection current density, $l_{k}$ and $I_{k}$ are the length and the pump current of the $k$-th laser section $S_{k}, \sigma$ is the cross-section area of the active zone, $A, B$ and $C$ are three coefficients of the cubic function describing the rate of non-radiative and radiative spontaneous recombination. 
The complex field propagation factor $\beta$ is defined as

$$
\begin{aligned}
& \beta=\delta(n, I)+\frac{i(g(n)-\alpha)}{2}, \quad \delta=\delta_{s}+\delta_{n}(n)+\delta_{T}(I), \\
& g(n)=g^{\prime} n_{t r} \ln \left(\frac{\max \left\{n, n^{*}\right\}}{n_{t r}}\right), \delta_{n}(n)=\tilde{n}^{\prime}\left(n-n_{t r}\right), \\
& \left.\delta_{T}\right|_{z \in S_{k}}=\delta_{T, k}(I)=\frac{2 \pi n_{g}}{\lambda_{0}^{2}} \sum_{r} \nu_{k}^{r} I_{r}, \quad r, k \in\{A, B R\} .
\end{aligned}
$$

Here, the parameters $\alpha$ and $\delta_{s}$ represent the internal absorption and the static detuning. $g^{\prime}$ and $\tilde{n}^{\prime}$ are the differentials of the carrier dependent effective logarithmic gain and linear index change functions $g(n)$ and $\delta_{n}(n)$ at the transparency carrier density $n_{t r}$. $n^{*}$ is the reference carrier density for limiting the absorption in the logarithmic gain function. The coefficients $\nu_{k}^{r}$ of the linear thermal detuning function $\delta_{T}(I)$ determine the impact of the injection currents $I_{r}$ on the refractive index change within each laser section $S_{k}[9] . \quad \lambda_{0}, c_{0}$ and $e$ are the central wavelength, the speed of light in vacuum and the electron charge, respectively. The values of the laser parameters used in our simulations and given in Table 1 were obtained in different ways. For example, the coupling coefficient and the internal absorption were obtained by a fit of the power-current characteristics at front and rear facets, see Fig. 6 . In doing so we neglected the fact that the internal quantum efficiency is less than $100 \%$. However, from an analysis of the length-dependence of the slope efficiency of similar broad-area devices we obtained values greater than $95 \%$ such that the internal absorption is only slightly overestimated. The cross-section of the active zone was calculated taking a total thickness of $21 \mathrm{~nm}$ of the three quantum wells and an effective width of the ridge waveguide of $10 \mu \mathrm{m}$ to account for lateral current spreading and carrier diffusion.

Owing to the high effective duty cycle of the sixth order grating $(>80 \%)$, the radiation losses are low and the imaginary part of the coupling coefficient can be neglected [3]. Other parameters (e.g. transparency carrier density, differential gain and index) were obtained from a microscopic gain model [15] or taken from the literature [16]. The impact of the waveguide structure is accounted for in the effective modal gain and index functions, with optical confinement factor of 0.022 .

\section{Mode analysis}

Before switching to simulations of the TW model (1)-(3) let us discuss the origin of the experimentally observed (see Figs. 2 and 3 ) periodically reappearing state transitions with the increase of the injection current. For this reason we perform an analysis of the instantaneous optical modes [10] which determines the different continuous wave states observed experimentally.

For any fixed carrier distribution $n(z)$ the optical field equations (1) and the assumption $E(z, t)=\Theta(\beta(n), z) e^{i \Omega(\beta(n)) t}$ give rise to a spectral problem defined by the following equations:

$$
\begin{aligned}
& \left(\begin{array}{cc}
-\partial_{z}-i D(\beta, \Omega) & -i \kappa \\
-i \kappa & \partial_{z}-i D(\beta, \Omega)
\end{array}\right)\left(\begin{array}{c}
\Theta^{+} \\
\Theta^{-}
\end{array}\right)=0, \\
& \Theta^{+}(\beta, 0)=r_{0} \Theta^{-}(\beta, 0), \quad \Theta^{-}(\beta, L)=r_{L} \Theta^{+}(\beta, L), \\
& D(\beta, \Omega)=\beta(n)+\frac{n_{g}}{c_{0}} \Omega-\chi(\Omega),
\end{aligned}
$$

where $\chi(\Omega)=i \frac{\bar{g}}{2} \frac{i(\Omega-\bar{\omega})}{\bar{\gamma}+i(\Omega-\bar{\omega})}$ is a frequency (wavelength) dependent contribution to the material gain dispersion. 
Table 1. Parameters used in simulations.

\begin{tabular}{llllc}
\hline & Description & Unit & $S_{A}$ & $S_{B R}$ \\
\hline$l$ & section length & $m m$ & 3 & 1 \\
$\kappa$ & coupling coefficient & $1 / \mathrm{cm}$ & 0 & 9.6 \\
$I$ & injection current & $A$ & {$[0-0.9]$} & 0 \\
$\nu^{A}$ & thermal detuning coef. & $n m / A$ & 0.75 & 0.2 \\
$\nu^{B R}$ & thermal detuning coef. & $n m / A$ & 0 & 0 \\
\hline$n_{g}$ & group refractive index & & 3.6 \\
$\alpha$ & internal absorption & $1 / \mathrm{cm}$ & 2.7 \\
$\delta_{s}$ & static detuning & $1 / \mathrm{cm}$ & -10 \\
$n_{t r}$ & transparency carrier density & $1 / m^{3}$ & $1.3 \cdot 10^{24}$ \\
$n^{*}$ & absorption limiting density & $1 / m^{3}$ & $0.95 \cdot 10^{24}$ \\
$g^{\prime}$ & effective differential gain & $\mathrm{m}^{2}$ & $7.26 \cdot 10^{-21}$ \\
$\tilde{n}^{\prime}$ & effective differential index & $\mathrm{m}^{2}$ & $-7.26 \cdot 10^{-21}$ \\
$\sigma$ & cross-section of active zone & $\mu m^{2}$ & 0.21 \\
$A$ & recombination parameter & $1 / n s$ & 0.4 \\
$B$ & recombination parameter & $m^{3} / \mathrm{s}$ & $1 \cdot 10^{-16}$ \\
$C$ & recombination parameter & $\mathrm{m}^{6} / \mathrm{s}$ & $1 \cdot 10^{-41}$ \\
$\bar{g}$ & Lorentzian gain amplitude & $1 / \mathrm{cm}$ & 50 \\
$2 \bar{\gamma}$ & Lorentzian FWHM & $1 / \mathrm{ps}$ & 100 \\
$\bar{\omega}$ & Lorentzian peak detuning & $1 / \mathrm{ps}$ & 2 \\
\hline$\lambda_{0}$ & central wavelength & $\mu m$ & 1.064 \\
$r_{0}$ & amplitude reflection (front facet) & & -0.1 \\
$r_{L}$ & amplitude reflection (rear facet) & & 0.01 \\
\hline & & &
\end{tabular}

The instantaneous, i.e., carrier dependent optical compound cavity modes are determined by the pairs $[\Theta(z), \Omega]$, where the eigenfunction $\Theta$ and the eigenvalue (complex frequency) $\Omega$ solve the spectral problem (4). The real and imaginary parts of $\Omega$ are the optical angular frequency and the damping of the mode, respectively. A continuous wave (cw) state of the system (1)-(3) is defined by an optical mode $[\Theta(\beta(\bar{n}), z), \Omega(\beta(\bar{n}))]$ with a real eigenvalue $\Omega$. Here, the carrier distribution $\bar{n}(z)$ fulfills the stationary carrier rate equation (2).

Let us consider some cw state of our TW model determined by functions $\bar{n}(z)$, $\Theta_{s}(\beta(\bar{n}), z)$ and (real) mode frequency $\Omega_{s}(\beta(\bar{n}))$. In general, a change of laser parameters (e.g., injection currents) implies some changes of this $\mathrm{cw}$ state. Below we present two simple parameter tuning mechanisms that maintain the carrier and photon distributions $\bar{n}(z)$ and $\left|\Theta_{s}(\beta(\bar{n}), z)\right|^{2}$ of this state.

The first mechanism makes use of a uniform change of the propagation factor $\beta$ in all sections by the same (real) factor $\Delta_{\beta}$. It follows from (4) that such modification preserves all eigenfunctions $\Theta(\beta, z)$ and shifts the corresponding eigenvalues $\Omega(\beta)$ of the spectral problem $(4): \ddagger$

$$
\Theta\left(\beta+\Delta_{\beta}, z\right)=\Theta(\beta, z), \quad \Omega\left(\beta+\Delta_{\beta}\right)=\Omega(\beta)-\frac{c_{0}}{n_{g}} \Delta_{\beta} .
$$

Since the mode frequencies $\Omega$ change by a real factor, the cw state frequency $\Omega_{s}$ remains real and the (modified) $s$-th mode $\left[\Theta_{s}(z), \Omega_{s}\right]$ defines a cw state again. This mode frequency tuning mechanism determines the slow basic red shift $\partial_{I} \lambda_{s}$ of the lasing wavelength (see Fig. 3).

$\ddagger$ We neglect small changes of the material gain dispersion for closely located frequencies, i.e., assume that $\left.\partial_{\xi} \chi(\xi)\right|_{\xi=\Omega}=0$. 
The second mechanism makes use of a shift of the detuning

$$
\delta_{k} \stackrel{\text { def }}{=} \frac{1}{l_{k}} \int_{S_{k}} \delta(n, I, z) d z, \quad k \in\{A, B R\}
$$

in the amplifying section $S_{A}$ alone. The field coupling coefficient $\kappa$ in this section vanishes and the eigenfunctions $\Theta$ are given by

$$
\Theta^{ \pm}(\beta, z)=\Theta^{ \pm}(\beta, 0) e^{\mp i \int_{0}^{z} D(\beta(s), \Omega) d s}, \quad 0 \leq z \leq l_{A} .
$$

The shift of the factor

$$
\varphi=l_{A} \delta_{A} / \pi
$$

by any integer number $m$ preserves the complex ratio between the mode components $\Theta^{+}$and $\Theta^{-}$at the amplifier/reflector interface $z=l_{A}$, and, therefore, the distributions $\Theta^{ \pm}(z)$ within the DBR section $S_{B R}$ (up to the multiplier $(-1)^{m}$ ) are also unchanged. Consequently, an integer shift of the phase factor $\varphi$ preserves the modal frequencies $\Omega$ and the eigenfunction intensity distributions $\left|\Theta^{ \pm}(z)\right|^{2}$. As it will be shown below, such change of $\varphi$ is responsible for the periodically reappearing lasing mode transitions and for the fast red shift $\partial_{I} \lambda_{f}$ of the lasing wavelength.

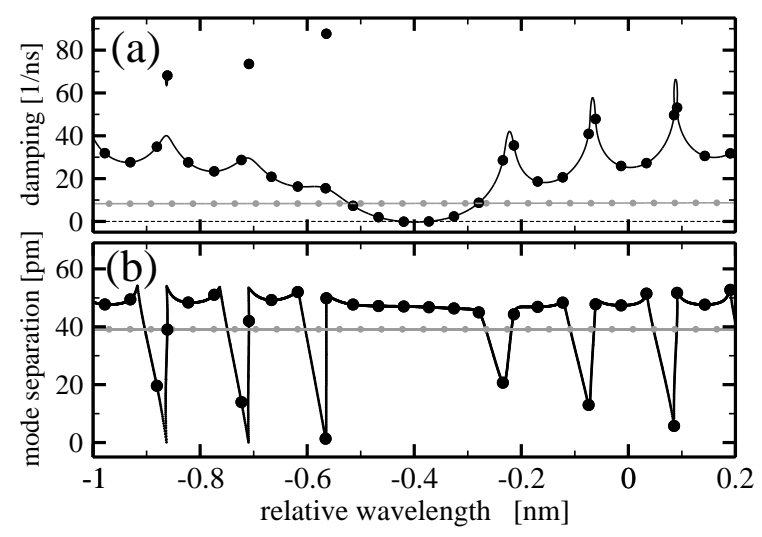

Figure 4. Spectral analysis of the DBR laser (black) and the reference FabryPerot cavity (gray). Symbols: complex eigenvalues (a) and wavelength separation of the eigenvalue to its closest short-wavelength neighbor (b). Lines: changes of the above mentioned quantities with the changing phase factor $\varphi$.

To get a deeper understanding of the periodic mode transitions we have performed a modal analysis [10] of the DBR laser under investigation. The evolution of the complex frequencies $\Omega$ computed with some fixed $n(z)$ and tuned phase factor $\varphi$ is summarized in Figs. 4 and 5. Black symbols in Fig. 4(a) show the location of these eigenvalues in the complex wavelength/damping plain. The black curves connecting these eigenvalues represent their shift with changing $\varphi$.

The eigenvalues moving along the curve are replacing their next neighbor after a 1-periodic shift of this factor. Note also three separated eigenvalues which move 1-periodic in $\varphi$ around some small closed loops. These separated loops are implied by the field coupling in the DBR section and can be connected to the main curve or shifted to the large damping values by an appropriate choice of the coupling strength 
or the field loss parameters. For more details on the formation of such loops see Refs. [10, 11].

A mode with zero damping determines the $\mathrm{cw}$ state of the device. The other 5-6 modes located within the stop band of the DBR can be excited by the spontaneous emission noise and, therefore, can be recognized in the optical spectra and the spectral mapping diagrams. To emphasize the mode selection by the Bragg grating we consider also the modes of a reference Fabry-Perot (FP) cavity with its length set equal to the total cavity length of the DBR laser (see gray bullets and lines in the same diagram). Another important feature of the DBR section is shown in Fig. 4(b). Here, the black symbols represent the wavelength separation of a mode from its closest shortwavelength neighbor, which is not necessarily located on the same curve of Fig. 4(a). In contrast to the reference FP cavity (grey bullets and lines), where a $\sim 39 \mathrm{pm}$ mode separation is strictly related to the field round-trip in the device, the mode separation in the DBR laser depends on the mode wavelength and the value of the phase factor $\varphi$ (see the different positions of the black bullets and the variation of the thin black curve). The minima of the mode wavelength separation for selected phases $\varphi$ occur at the sides of the DBR stop band and are related to the separate small closed loops (shorter-wavelength side of Fig. 4(a)) or the sharp peaks of the open black curve (longer-wavelength side of the same diagram). At the shorter-wavelength side a pair of eigenvalues belonging to some small closed loop and the open curve can have the same wavelength but different damping. At the longer-wavelength side the minimal wavelength separation is achieved when a pair of eigenvalues are located on the opposite sides of the same sharp peak of the open curve. We note, however, that being outside of the stop band these modes are well damped and are not relevant for the operation of the DBR laser.

The separation of the least damped modes at the stop band of the DBR $(\sim 47$ $\mathrm{pm}$ in our case) is larger than the $\sim 39 \mathrm{pm}$ mode separation of the reference FP cavity. This is in a nice agreement with the experimentally observed $\sim 45 \mathrm{pm}$ mode separation visible in Fig. 3. The increase of the mode separation within the stop-band of the DBR can be explained by the decrease of the effective DBR (and the compound cavity) length for the monochromatic fields with the corresponding optical frequencies.

Figure 5 gives another representation of the spectral analysis from Fig. 4. Here we show how the damping (panel a) and the wavelength (panel b) of several most important DBR stop-band modes change with the phase parameter $\varphi$. Due to periodicity of this parameter, the curves leaving these two diagrams at the right side $(\varphi=1)$ reenter the diagrams from the left side $(\varphi=0)$.

While the black curves in panel (a) represent only a small change in the main mode damping, the nearly straight and almost parallel curves in panel (b) indicate an almost linear increase of the modal (and lasing) wavelength with the changing factor $\varphi$ which is nearly proportional to the injection current into amplifying sections. This linear increase of the modal wavelengths corresponds to the difference between experimentally observed periodically reappearing fast red shift and slow red shift of the lasing wavelengths (see Fig. 3).

The transitions between the modes seen in Fig. 3 occur when the damping of the neighboring mode becomes smaller than the zero damping of the mode corresponding to the $\mathrm{cw}$ operation of the laser. A similar situation (even though we assumed a carrier distribution $n$ that is time invariant) can be recognized in Fig. 5(a). The exchange between the dominant modes represented by bullets and squares is expected to occur close to the value of $\varphi$ indicated by the vertical dashed line. 


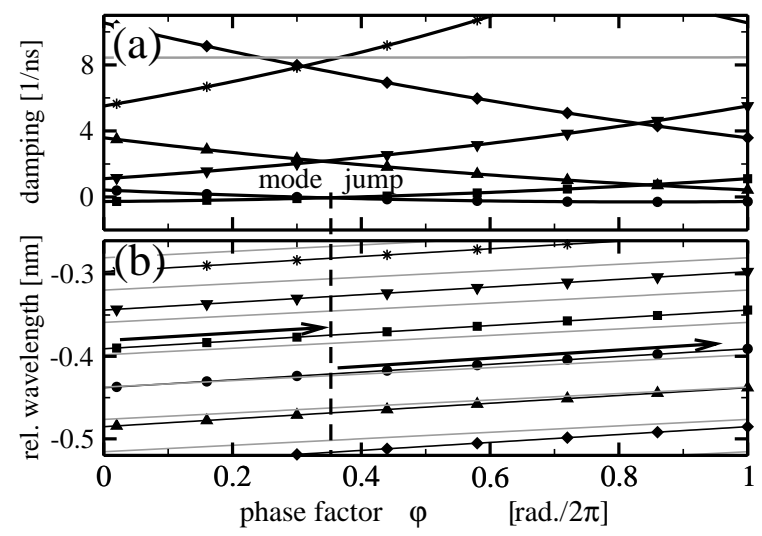

Figure 5. Changes of the damping (a) and the wavelength (b) of several of the most important modes (indicated by symbols) with the changing phase factor $\varphi$. The arrows and the vertical dashed line indicate the dominant modes with the lowest damping and the position where transition between these modes can be expected. Gray lines represent the reference Fabry-Perot cavity.

The grey lines in Fig. 5 represent a reference FP cavity where the damping of the modes is almost independent on the mode number or phase factor $\varphi$ (see panel a). The mode wavelengths are equidistant and change linearly with the factor $\varphi$ (see panel b). The mode separation is different from that of the DBR laser (see discussion above). Consequently, the expected (fast) red shift of the wavelengths in this FP cavity is smaller than that one of the DBR laser.

Taking into account the fact that the detuning $\delta$ changes in all laser sections simultaneously, we conclude:

- The slow red shift of the lasing wavelength

$$
\partial_{I} \lambda_{s} \approx\left(\lambda_{0}^{2} / 2 \pi n_{g}\right) \partial_{I_{A}} \delta_{B R}
$$

observed experimentally occurs due to the changing detuning $\delta_{B R}$.

- The periodicity of the mode transitions with the increasing injection current in the amplifier section can be approximated by

$$
I_{\text {per }}=1 / \partial_{I_{A}} \tilde{\varphi}, \quad \tilde{\varphi}=\varphi-l_{A} \delta_{B R} / \pi .
$$

That is, the periodic mode transitions occur due to the nearly linear increase of the relative index change function $\tilde{\varphi}$.

- The fast red shift of the lasing wavelength can be approximated by

$$
\partial_{I} \lambda_{f} \approx \partial_{I} \lambda_{s}+\Delta_{\lambda} / I_{\text {per }},
$$

where $\Delta_{\lambda}$ denotes the separation between the wavelengths of the two dominant modes involved in the transition.

The mode analysis presented reproduces the experimentally observed mode transitions well and allows us to estimate some of the coefficients $\nu_{k}^{r}$ entering the thermal detuning model $\delta_{T}$ in (3). In order to determine $\nu_{k}^{r}$ we first estimate the slow wavelength shift $\partial_{I} \lambda_{s}$ and the mode transition period $I_{\text {per }}$ from Fig. 3, and assume that the impact of electronic detuning $\delta_{n}$ to the changes of the full detuning $\delta$ is small 
to give the relationship:

$$
\partial_{I_{r}} \delta_{k} \approx \partial_{I_{r}} \delta_{T, k}=\frac{2 \pi n_{g}}{\lambda_{0}^{2}} \nu_{k}^{r}, \quad k, r \in\{A, B R\}
$$

Using this relationship in combination with (6) and (7) allows us to estimate $\nu_{A}^{A}$ and $\nu_{B R}^{A}$.

Due to the fact that the DBR section is unbiased $\left(I_{B R}=0\right)$, the injection current $I_{B R}$ has no influence to the thermal detuning function $\delta_{T}$, and, therefore, to the shift of the mode wavelengths or to the jumps between different lasing states. Thus, we can assume $\nu_{A}^{B R}=\nu_{B R}^{B R}=0$ which completes the definition of the thermal detuning function $\delta_{T}$.

\section{Simulations}

In this section we present our simulation results and discuss some parameter fitting procedures.

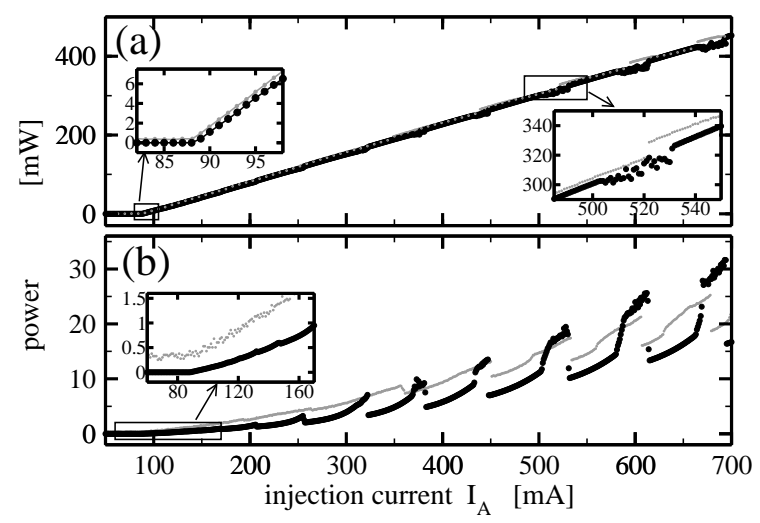

Figure 6. Power-current characteristics of the DBR laser at the front (a) and rear (b) facets. Black: theory. Gray: experiments.

Figure 6 shows simulated and experimental power-current characteristics of the considered DBR laser. Several basic features such as

- periodically occurring undulations of the emitted power,

- transitions characterized by a sudden increase (decrease) of the power emitted from the front (rear) facets and

- a non-monotonous growth of the power emitted from the rear facet (typical for non-stationary lasing states) before state transitions

are properly reproduced by our simulations. The slope efficiency at both facets of the laser is comparable to experiments and was obtained by a careful choice of the field coupling coefficient $\kappa$ in the DBR section and the internal absorption.

The lasing threshold was fitted mainly by the choice of the absorption limiting density $n^{*}$. The suggested limitation of the gain function for small carrier densities is in agreement with simulations of a microscopic gain model which reveal an initial slight increase and then a decrease of the absorption with increasing carrier density, in 


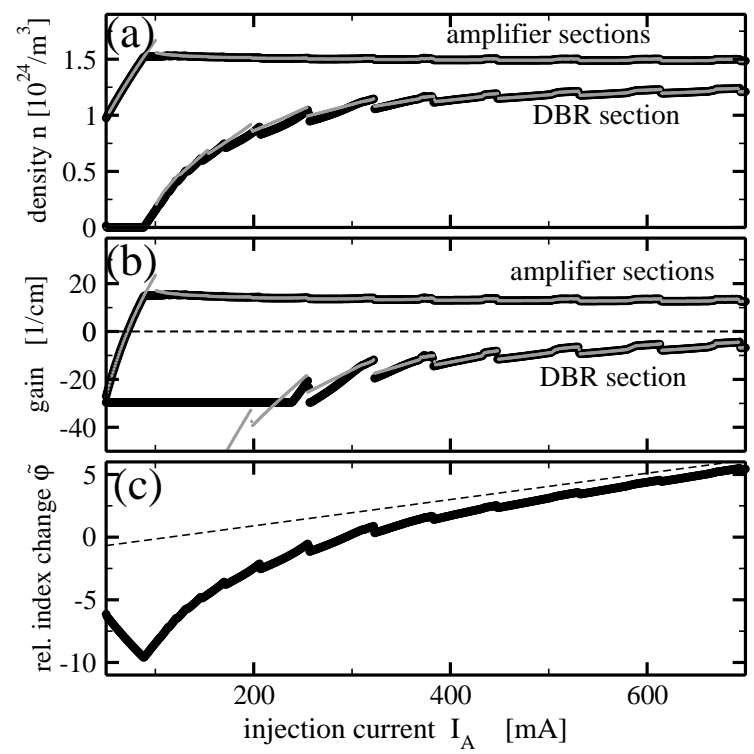

Figure 7. Simulated mean carrier density $n$ (a), gain/loss function $g(n)(\mathrm{b})$ and the relative index change function $\tilde{\varphi}$ from equation (7) (c) as a function of the injection current $I_{A}$. The dashed line in panel (c) is the function $\tilde{\varphi}$ with corrections due to the electronic detuning $\delta_{n}$ neglected. Black and grey: absorption limited and non-limited $\left(n^{*}=0\right)$ gain models.

dependence on the wavelength [17]. For $n^{*}=0$ the modeled field absorption $\alpha-g(n)$ in the unpumped DBR section in the below threshold regime is un-physically high, which would lead to an increase of the theoretical threshold current by, approximately, $10 \%$. The influence of a non-zero $n^{*}$ at small injection currents is illustrated by comparing thick black lines (absorption limited model) and thin gray (non-limited model) lines in Fig. 7(b). The simulated carrier density values, however, in both these approaches are approximately the same (see panel (a) of the same figure).

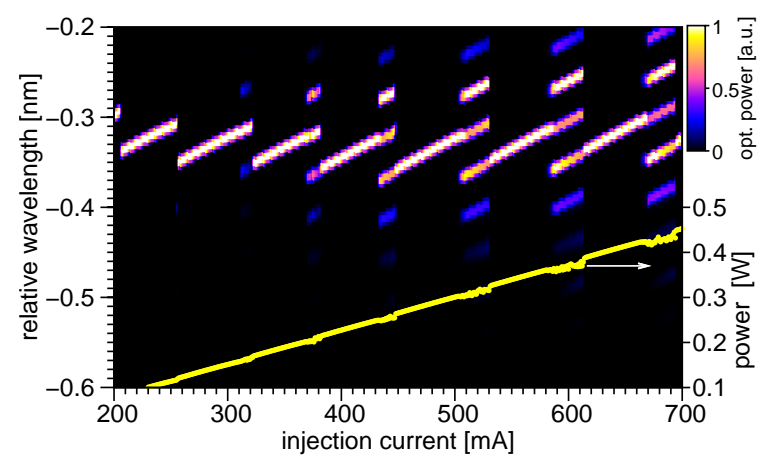

Figure 8. Representation of the simulated optical spectra (mapping) and the emitted power (light curve) at the front facet of the laser as a function of the increased injection current.

In Fig. 8 the change of the simulated optical spectrum with the increase of $I_{A}$ is 
presented. As seen in experiments (see figure 3), we observe here periodically occurring jumps to a short-wavelength neighboring mode in the optical spectra, a multiple peak optical spectra before and a single mode emission after the state transitions. The mode separation in simulations is $\sim 47 \mathrm{pm}$, which is comparable to the experiments and is significantly larger than the mode separation in a similar FP-type laser. Finally, in both theoretical and experimental cases the wavelength shift within a single "period" of injection current is nearly linear.

Although our simulations successfully reproduce most experimental observations, Figs. 3 and 8 also show us several discrepancies. Compared to experiments, in simulations we have

- a much more regular periodicity of mode transitions, and

- a less pronounced general slow red shift for larger $(>400 \mathrm{~mA})$ and a blue shift for smaller $(<400 \mathrm{~mA})$ currents.

The first discrepancy is due to our assumption of the linear thermal detuning model. In practice, different experimental mode transition periods correspond to different thermal detuning factors $\nu$ in (3).

The second of these effects in our simulations is due to the fast growth of $n$ in the DBR section at small above-threshold currents as seen in the lower black curve in Fig. 7(a). For these injection currents, a (blue) carrier density driven shift in the refractive index $\delta_{n}(n)$ in the DBR section plays a significant role. It counteracts and even exceeds the thermal red shift $\delta_{T}(I)$ in the DBR section, that in turn results in a general blue wavelength shift visible for small $I_{A}$ in Fig. 8.

Another effect of this non-vanishing $\delta_{n}(n)$ is represented by differences in the periods of the mode transitions. The derivation of the heating parameters $\nu$ from the experimentally estimated slow red shift $\partial_{I} \lambda_{s} \approx 20 \mathrm{~nm} / \mathrm{A}$ and the transition period $I_{\text {per }} \approx 95 \mathrm{~mA}$ in Section 4 was made by neglecting the contribution of $\delta_{n}$. For small above-threshold currents the electronic detuning $\delta_{n}(n)$ in the amplifying sections changes only weakly, while in DBR section it decays significantly. This in turn implies an additional positive shift of $\tilde{\varphi}(I)$ which can be even larger then the thermal one: see, for illustration, the slopes of the thin dashed and thick black lines in Fig. 7(c) representing a pure thermal and a full detuning, respectively. The growth of the function $\tilde{\varphi}(I)$ at lower $I_{A}$ is much larger than at high $I_{A}$. Since a current interval between a consequent mode switching corresponds to the change of $\tilde{\varphi}(I)$ by 1 , these intervals are much shorter for small injection currents. The elimination of the general blue shift and the increase of the mode-jumping periods at small injection currents could be achieved by a modification of the carrier dependent index change function $\delta_{n}(n)$ so that $\partial_{n} \delta_{n} \approx 0$ at small $n$.

For increased amplifier currents the intensity of the optical field entering DBR section grows. The balance of the carrier rate equation (2) in this section is kept by increasing the gain (decreasing the loss) function $g(n)<0$, i.e., by increasing the carrier density, which for high injection levels slowly approaches the transparency density $n_{t r}$ (see Fig. 7a). Due to a slow change of $n$ at larger currents the contribution of $\delta_{n}$ is by an order of magnitude smaller than that of $\delta_{T}$, and the $\approx 84 \mathrm{~mA}$ simulated mode transition period here is only $\sim 10 \%$ smaller than initially assumed one. 


\section{Conclusions}

We investigated experimentally and numerically the electro-optical behavior of a multisection DBR semiconductor laser with an active region extending over all sections. The laser emits an optical power of $600 \mathrm{~mW}$ at a current of $900 \mathrm{~mA}$. The power-current characteristics show nearly periodically reappearing jumps, where the power at the front facet increases and the power at the rear facet decreases. With an increase of the injection current the laser device exhibits an average slow shift as well as periodically reappearing fast red shifts of the lasing wavelength. The corresponding periodic jumps to adjacent short-wavelength cavity modes are connected with pulsations of the field intensity.

The modal analysis and numerical simulations reveal the mechanisms of the periodic mode jumps, the observed dynamic regimes and the slow or fast shifts of the lasing wavelength. The periodicity of the mode transitions with an increase of injection current is caused by a difference in the refractive index tuning rates in DBR and amplifying sections. The observed 3 stage sequence (1) cw operation, (2) mode-beating pulsations and (3) abrupt change of the lasing wavelength is a direct consequence of the change of gain and damping of the modes with the change of refractive indices. Here, mode-beating pulsations and subsequent mode jumping are due to the vanishing damping of the neighboring mode which eventually becomes the new dominant mode.

The average slow shift of the lasing wavelength arises from the dependencies of the refractive index in the DBR section on the local carrier density and temperature. At low power the increase of the carrier density due to optical pumping can dominate over the thermal detuning and lead to an average blue shift of the lasing wavelength. At higher power the carrier density in optically well-pumped DBR section becomes saturated, which reduces the impact of the electronic detuning with increasing injection current. In this high-power regime, the variation of the total detuning in DBR section is now dominated by the increase of the temperature due to cross-heating from the electrically pumped sections, leading to an average red shift of the lasing wavelength.

The fast shift is determined by the separation of the neighboring cavity modes, by the length of the mode-transition period and, finally, by the slow wavelength shift rate. By choosing proper dependencies of the refractive index in each section on the currents injected into the same and the other section good agreement between experiment and simulation was obtained.

Many applications require stable lasing of a single longitudinal mode under different operational conditions (pulsed, modulated, cw) over a wide range of parameters such as currents and temperatures. For the case of $\mathrm{cw}$ operation considered in this paper one would be interested in an increase of the spacing between the mode jumps and a decrease of the dynamic mode transition regions. According to our analysis, the first task could be achieved by decreasing the length of the amplifier, by other modifications of the device leading to an appropriate change of heating coefficients $\nu$, or by a simultaneous tuning of the injection currents in the DBR [18] or some additional passive [19] or active sections. The reduction of the dynamic mode transition regions can be achieved by, e.g., an optimization of front facet reflectivity, coupling coefficient and length of DBR section: see a related study of DFB lasers in Ref. [11]. Both these optimization procedures, however, are subject to a further study. 


\section{Acknowledgments}

The work of M.R. was supported by DFG Research Center MATHEON "Mathematics for key technologies: Modelling, simulation, and optimization of real-world processes". H. W. thanks C. Schwanke for support in the numerical simulation.

\section{References}

[1] Wenzel H, Fricke J, Klehr A, Knauer A and Erbert G 2006 IEEE Photon. Techn. Lett. 18 737-9

[2] Nguyen H K, Coleman S, Visovsky N J, Li Y, Song K, Davis R W, Hu M H and Zah C 2007 Electron. Lett. 43 716-7

[3] Fricke J, Wenzel H, Matalla M, Klehr A and Erbert G 2005 Semicond. Sci. Technol. 20 1149-52

[4] Price R K, Borchardt J J, Elarde V C, Swint R B and Coleman J J 2006 IEEE Photon. Techn. Lett. 18 97-9

[5] Achtenhagen M, Amarasinghe N V and Evans G A 2007 Electron. Lett. 43 755-7

[6] Bandelow U, Radziunas M, Sieber J and Wolfrum M 2001 IEEE J. Quantum Electron. 37 183-8

[7] Hofstetter D and Zappe H P 1997 Appl. Phys. Lett. 71 181-3

[8] Achtenhagen M, Amarasinghe N V, Jiang L, Threadgill J and Young P 2009 J. Lightwave Techn. $273433-7$

[9] Spreemann M, Lichtner M, Radziunas M, Bandelow U and Wenzel H 2009 IEEE J. Quantum Electron. 45 609-16

[10] Piprek J (ed) 2005 Optoelectronic Devices: advanced simulation and analysis (New York: Springer) pp 121-50.

[11] Bauer S, Brox O, Kreissl J, Sartorius B, Radziunas M, Sieber J, Wünsche H-J and Henneberger F 2004 Phys. Rev. E 69, 016206

[12] Radziunas M, Tronciu V Z, Bandelow U, Lichtner M, Spreemann M and Wenzel H 2008 Opt. and Quant. Electron. 40 1103-9

[13] Wenzel H, Bugge F, Dallmer M, Dittmar F, Fricke J, Hasler K-H and Erbert G 2008 IEEE Photon. Techn. Lett. 20 214-16

[14] Hasler K-H, Sumpf B, Adamiec P, Bugge F, Fricke J, Ressel P, Wenzel H, Erbert G and Tränkle G 2008 IEEE Photon. Techn. Lett. 20 1648-50

[15] Wenzel H, Erbert G and Enders P 1999 IEEE J. Select. Topics Quant. Electron. 5 637-42

[16] Piprek J 2003 Semiconductor Optoelectronic Devices: Introduction to Physics and Simulation (Amsterdam: Academic Press)

[17] Wenzel H, Klehr A, Erbert G, Sebastian J, Tränkle G, Pereira M F 2000 Appl. Phys. Lett. 76 2653-55

[18] Hu M H, Nguyen H K, Song K, Li Y, Visovsky N J, Liu X, Nishiyama N, Coleman S, Hughes Jr. L C, Gollier J, Miller W, Bhat R and Zah C-E 2006 Optical Components and Materials III, Proc. SPIE 6116 61160M1-8

[19] Kitaoka Y, Kasazumi K, Morikawa A, Yokoyama T, Sugita T, Mizuuchi K, Yamamoto K, Takayama T, Takigawa S and Yuri M 2003 Optics Letters 28 914-16 